University of Nebraska - Lincoln

DigitalCommons@University of Nebraska - Lincoln

Norman R. Simon Papers

Research Papers in Physics and Astronomy

$5-1-1986$

\title{
Hydrodynamic Models Of RRab Variables: Comparison Of Observed And Theoretical Light Curves
}

Norman R. Simon

University of Nebraska - Lincoln, nsimon@unl.edu

Follow this and additional works at: https://digitalcommons.unl.edu/physicssimon

Simon, Norman R., "Hydrodynamic Models Of RRab Variables: Comparison Of Observed And Theoretical Light Curves" (1986). Norman R. Simon Papers. 21.

https://digitalcommons.unl.edu/physicssimon/21

This Article is brought to you for free and open access by the Research Papers in Physics and Astronomy at DigitalCommons@University of Nebraska - Lincoln. It has been accepted for inclusion in Norman R. Simon Papers by an authorized administrator of DigitalCommons@University of Nebraska - Lincoln. 
The Astrophysical Journal, Vol. 160, June 1970

(C) 1970. The University of Chicago. All rights reserved. Printed in U.S.A.

\title{
INFLUENCE OF OPACITY ON THE PULSATIONAL STABILITY OF MASSIVE STARS WITH UNIFORM CHEMICAL COMPOSITION
}

\author{
Richard Stothers and Norman R. Simon \\ Institute for Space Studies, Goddard Space Flight Center, NASA, New York \\ Received 1969 October 6; revised 1969 November 28
}

\section{ABSTRACT}

The maximum mass of homogeneous stars which are stable against nuclear-energized pulsations has been redetermined by using a full opacity formula and an accurate treatment of the equilibrium structure of the outer layers where nonscattering sources of opacity are important damping agents. Sixteen composition mixtures were used, covering the range $0 \leq Y \leq(1-Z)$ and $0 \leq Z \leq 0.05$. The nuclearenergy sources were taken to be the $\mathrm{CN}$ cycle and the triple- $a$ process. Due to the primary influence of bound-free absorption by metals (and, to a lesser extent, of free-free absorption by hydrogen and helium), the critically stable nondimensional mass of hydrogen-burning stars, $\left(M / M_{\odot}\right) \mu^{2}$, can range from 27 to 94 , depending on the chemical composition. For likely chemical compositions of extreme Population I, the actual critical mass is probably $80-120 M_{\odot}$. For homogeneous helium-burning stars, the critical mass ranges from 13 to $19 M_{\odot}$, depending on the metal abundance.

\section{INTRODUCTION}

The pulsational stability of stars against nuclear-energy release was first discussed in 1918 by Eddington, even though at that time the existence of the nuclear sources was not yet proved. Subsequent developments up to Ledoux's important investigation of 1941 have been summarized by Rosseland (1949), while more recent work, beginning with that of Ledoux, is abstracted for convenience in Table 1 in the case of chemically homogeneous stars of high mass.

One very important factor which has been largely neglected so far is the opacity source; this is the main subject of the present paper. Boury (1964) and Noels-Grötsch (1967) have, indeed, considered the effect of introducing Compton scattering in place of the customarily adopted Thomson scattering. However, this relatively small correction will be replaced in the present paper by a complete opacity formula which takes into account sources of atomic absorption as well. Since atomic absorption is important only in the outer envelope of the star (where most of the pulsational damping occurs), a treatment of the structure of the surface layers that is more accurate than that yielded by the zero boundary conditions heretofore used is also in order. Finally, the maximum mass for pulsational stability will be determined for a broad range of homogeneous models, constituted principally of hydrogen and helium (in differing ratios) with the metals abundance taken to be $0 \leq Z \leq 0.05$.

\section{ASSUMPTIONS}

The basic equilibrium equations for the structure of chemically homogeneous stars have been written in complete form by Schwarzschild (1958), while the basic perturbation equations for small radial pulsations have been written in linearized, adiabatic form by Schwarzschild and Härm (1959). The problem is to determine, for a selected chemical composition, the critical mass at which the net gain or loss rate of pulsational energy over a cycle vanishes, viz., $L_{P}{ }^{*}=0$, in the notation of Schwarzschild and Härm (but where the asterisk denotes our omission of running waves at the surface). The following subsections are devoted to the physical parameters needed to evaluate this quantity and to the adopted modifications of the procedure of Schwarzschild and Härm. 
TABLE 1

Previous Determinations of The Maximum Stable Mass of Homogeneous Stars

\begin{tabular}{|c|c|c|c|c|c|c|}
\hline$M / M_{\odot}$ & $\begin{array}{l}\text { Chemical } \\
\text { Composition }\end{array}$ & Model & $\begin{array}{c}\text { Radiation } \\
\text { Pressure }\end{array}$ & Opacity & $\begin{array}{l}\text { Energy Generation } \\
\text { in the Core }\end{array}$ & Author \\
\hline $\begin{array}{r}700-1200 \ldots \ldots \ldots \\
\sim 280 \ldots \ldots \ldots\end{array}$ & $\begin{array}{l}X \approx 1, Y \approx 10^{-4} \\
X \approx 1, \text { traces of } \mathrm{He}, \mathrm{C}, \mathrm{N}\end{array}$ & $\begin{array}{l}\text { Standard } \\
\text { Cowling }\end{array}$ & $\begin{array}{l}\text { Yes } \\
\text { Yes }\end{array}$ & $\begin{array}{l}\text { Thomson } \\
\text { Thomson }\end{array}$ & $\begin{array}{l}p-p \text { chain } \\
p-p \text { chain and CN } \\
\text { cycle }\end{array}$ & $\begin{array}{l}\text { Ledoux and Boury } 1960 \\
\text { Boury } 1963\end{array}$ \\
\hline$\sim 260$. & $X \approx 1$, traces of $\mathrm{He}, \mathrm{C}, \mathrm{N}$ & Cowling & Yes & Thomson & $\begin{array}{l}p-p \text { chain, } \mathrm{CN} \text { cycle, } \\
\text { and convection }\end{array}$ & Boury et al. 1964 \\
\hline$>280 \ldots \ldots \ldots$ & $X \approx 1$, traces of $\mathrm{He}, \mathrm{C}, \mathrm{N}$ & Cowling & Yes & Compton & $\begin{array}{l}p-p \text { chain and } C N \\
\text { cycle }\end{array}$ & Boury 1964 \\
\hline $\begin{array}{l}7-8 \ldots \ldots \\
9.2 \ldots \ldots\end{array}$ & $\begin{array}{l}Y=1 \\
Y=1\end{array}$ & $\begin{array}{l}\text { Cowling } \\
\text { Cowling }\end{array}$ & $\begin{array}{l}\text { Yes } \\
\text { Yes }\end{array}$ & $\begin{array}{l}\text { Thomson } \\
\text { Compton }\end{array}$ & $\begin{array}{l}3 a \text { process }(\nu=24) \\
3 a \text { process }(\nu=24)\end{array}$ & $\begin{array}{l}\text { Boury and Ledoux } 1965 \\
\text { Noels-Grötsch } 1967\end{array}$ \\
\hline
\end{tabular}




\section{a) Thermodynamics}

The equation of state is represented accurately throughout our models by the sum of perfect-gas pressure and radiation pressure, with the mean molecular weight of the gas given by the appropriate expression for a completely ionized gas. In our models hydrogen and helium are completely ionized out to the stellar surface.

\section{b) Opacity}

The opacity has been expressed as an analytical formula in terms of $\rho, T, X, Y$, and $Z$, in accordance with the precepts of Christy (1966), who fitted his own formula from tables of opacities similar to those published by Cox and Stewart (1965). We have found that Christy's formula works very well for the temperature-density conditions in our models except at high temperatures, where two improvements have been made. The first is the replacement of the formula for Thomson scattering by an approximate expression for Compton scattering (Deinzer and Salpeter 1965). The second is a more accurate representation of the contribution from bound-free absorption by metals (Morris and Demarque 1966). The complete opacity formula is

$$
\begin{aligned}
\kappa= & P_{E}\left[4.85 \times 10^{-13}\left[\rho T_{4}\left(1+2.2 \times 10^{-5} T_{4}\right)\right]^{-1}\right. \\
& +X\left\{T_{4}^{1 / 2}\left(2 \times 10^{6} T_{4}^{-4}+2.1 T_{4}^{6}\right)^{-1}+\left[4.5 T_{4}^{6}+T_{4}^{-1}\left(4 \times 10^{-3} T_{4}^{-4}\right.\right.\right. \\
& \left.\left.\left.+2 \times 10^{-4} \rho^{-1 / 4}\right)^{-1}\right]^{-1}\right\}+Y\left[\left(1.4 \times 10^{3} T_{4}+T_{4}^{6}\right)^{-1}\right. \\
& \left.+1.5\left(10^{6}+0.1 T_{4}^{6}\right)^{-1}\right]+Z\left\{T _ { 4 } ^ { 1 / 2 } \left[20 T_{4}+35 T_{4}^{4}\right.\right. \\
& \left.\left.\left.+18(1+X)^{0.33} \rho^{0.33} T_{4}^{4.71}\right]^{-1}\right\}\right]
\end{aligned}
$$

where $T_{4}=T \times 10^{-4}{ }^{\circ} \mathrm{K}$ and $P_{E}$ is the electron pressure. At all points, we have found that $P_{E}$ can be accurately represented by the proper expression for a completely ionized gas.

The four successive terms inside the double bracket for $\kappa$ represent essentially the following contributions: (1) scattering by free electrons, (2) free-free absorption by hydrogen, (3) free-free absorption by helium, and (4) bound-free and bound-bound absorption by metals. Christy has discussed in detail the form of the opacity formula.

Using typical runs of temperature and density from our models, we have checked the opacity formula against the Cox-Stewart (1965) tables for several normal and extreme choices of chemical composition in the ranges $0.009 \leq Y \leq 0.999,0.001 \leq$ $Z \leq 0.044$. Even in the worst cases (temperatures below $2 \times 10^{5}{ }^{\circ} \mathrm{K}$ ) the errors were always less than 30 percent, the estimated error of the tabulated opacities according to Cox and Stewart. For temperatures above $2 \times 10^{6}{ }^{\circ} \mathrm{K}$, the errors were less than 10 percent, becoming completely negligible at the temperatures characteristic of the convective-core boundary of our models, where electron scattering dominates.

The great advantages in our work of using a formula rather than a table of opacity are that the formula is more convenient to use if a variety of chemical compositions is being introduced, and that the formula provides a smooth opacity as a function of temperature and density, which is helpful when computing the thermodynamic derivatives of the opacity in the pulsation equations.

By writing $\kappa=\kappa_{0} \rho^{a} T^{\sim \eta}$ over a small range of density and temperature, the perturbed equation of radiative transfer can be expressed as

$$
\frac{\delta L(r)}{L(r)}=4 \frac{\delta r}{r}-a \frac{\delta \rho}{\rho}+(4+\eta) \frac{\delta T}{T}+T \frac{d}{d T}\left(\frac{\delta T}{T}\right)
$$


where $d / d T$ refers to the spatial run of temperature. It is simple to evaluate $a$ and $\eta$ from the thermodynamic derivatives of equation (1). In view of the coarseness of the grid of the tabulated opacities, it is impossible to estimate the error in $a$ and $\eta$, which critically affect the calculation of damping in the envelope. But we may hope that in the hot, adiabatic regions, which contribute most to the damping in our models, the opacities and the opacity exponents are reasonably well determined.

\section{c) Nuclear-Energy Generation}

In the homogeneous hydrogen-burning stars, the simple $\mathrm{CN}$ cycle operates because ${ }^{16} \mathrm{O}$ requires a long time to reach equilibrium. Therefore we have adopted $X_{\mathrm{CN}}=\frac{1}{3} Z$, which is the approximate fractional abundance of carbon and nitrogen derived from analyses of early-type stars (Traving 1966).

By writing $\epsilon=\epsilon_{0} \rho^{\lambda} T^{\nu}$, we find that the equilibrium rate of nuclear-energy generation is represented adequately as follows (Reeves 1965):

$$
\epsilon=10^{-91.3} X\left(\frac{1}{3} Z\right) \rho T^{13} .
$$

Since, over the range of central temperatures and of temperature profiles for our core models, $\nu$ varies by only \pm 1 , we have adopted uniformly $\nu=13$. Changing $\nu$ by a unit affects only the third significant figure of computed quantities in the equilibrium models.

The perturbed rate of nuclear-energy generation may be written in terms of effective exponents of the density and temperature as follows:

$$
\frac{\delta \epsilon}{\epsilon}=\lambda_{\text {eff }} \frac{\delta \rho}{\rho}+\nu_{\text {eff }} \frac{\delta T}{T} .
$$

Phase delays in the hydrogen-burning rate during the course of a pulsation have been approximately treated by following a standard prescription (e.g., Cox 1955; Ledoux and Boury 1960). We find that $\lambda_{\text {eff }}=1$ and that $\nu-\nu_{\text {eff }}$ lies in the range 0.6-3.0. For simplicity, we have set $\nu_{\text {eff }}=\nu=13$.

In the helium-burning models, the triple- $a$ process is adopted as an exclusive source of energy since the contributions from a-particle captures on carbon and heavier elements are very small. The equilibrium rate of energy generation may be written as (Reeves 1965)

$$
\epsilon=10^{-156.5} Y^{3} \rho^{2} T^{19} .
$$

This expression represents adequately the rate in the cores of all our helium-burning models. The effective exponents of the perturbed rate have been investigated by Perdang (1965), who finds that $\lambda_{\text {eff }}=2$ and $\nu_{\text {eff }}=\nu$, to an excellent approximation. Therefore, we are justified in setting $\nu_{\text {eff }}=19$. Neutrino losses due to the direct electronneutrino weak interaction have been shown to be negligible in massive helium-burning stars (Cimino et al. 1964; Masevich et al. 1965).

\section{d) Convection}

Convection in the stellar core has been treated as fully adiabatic. In the models with the highest helium content, a thin convective region occurs just below the photosphere due to the behavior of the helium opacity at low temperature; for simplicity, the equilibrium structure of such a region has been treated as fully adiabatic.

In the pulsation equations, the luminosity amplitudes of the convective core were computed by means of an average involving quantities evaluated at the center and the boundary of the core only (cf. Schwarzschild and Härm 1959). The adiabatic luminosity amplitudes in the thin convection zone at the surface of our models (which never covered 
more than 2 percent of the radius) were calculated by ignoring the convection entirely and by adopting the perturbed equation of radiative transfer (but see $\S$ II $f$ ).

\section{e) Surface Boundary Conditions}

The outermost layers of the star have been found to be in radiative equilibrium. The Eddington approximation has been adopted for the temperature structure, and an accurate integration of the equation of hydrostatic equilibrium has been performed for the pressure structure. As usual, we define the photosphere as the layer where the temperature is equal to the effective temperature of the star.

The boundary conditions for the pulsation equations have been derived by adopting adiabaticity of the thermodynamic equations out to the photosphere and by requiring regularity of the mechanical equation at $r=\infty$. (All the pulsation amplitudes above the photosphere are assumed to be constant.) Thus, in the latter case, the following equation applies:

$$
\left(\frac{\delta P}{P}\right)_{R}=-\left(4+\omega^{2}\right)\left(\frac{\delta r}{r}\right)_{R}
$$

where $\omega^{2}=(2 \pi / \text { Period })^{2} R^{3} / G M$. Baker and Kippenhahn (1965) have discussed other possible atmospheric boundary conditions.

\section{f) Nonadiabatic Effects in Outer Envelope}

The question of nonadiabatic regions is usually not discussed for pulsations in hot stars, and indeed is not important so long as the opacity is taken as due to scattering only. However, with the more realistic opacity used here, the adiabatic approximation leads to a region of unusually strong damping very near the surface. In the hydrogenburning models, this surface region is composed of two subzones: an upper zone with positive damping just below the photosphere, and a deeper zone with strong negative damping. In the helium-burning models, only the zone with negative damping appears. This negative damping is due primarily to the characteristics of the helium opacity which dominates at low temperatures, since the essentially Kramers-like form of the opacity due to metals which dominates at much higher temperatures in the deeper interior provides a normal positive damping.

According to a procedure described in the Appendix, it is found that the surface region in the hydrogen-burning models lies within the nonadiabatic layers where the flux is frozen in, and therefore contributes negligibly to the overall damping of the star. Since the subzones of positive and negative damping which appear in the adiabatic calculation cancel each other completely, we have, for simplicity, ignored the nonadiabatic effects entirely in determining the critical masses for the hydrogen-burning models. On the other hand, no such convenient cancellation occurs in the surface region of the helium-burning models, and we have therefore resorted to the nonadiabatic procedure outlined in the Appendix, which considerably modifies the apparently strong negative damping yielded by the adiabatic calculation. We might note here that the region of apparent negative damping lies wholly within the nonadiabatic layers where the flux is frozen in.

\section{CRITICAL MODELS}

A summary of the results of our calculations is presented in Tables 2 and 3, which contain the interpolated characteristics for models with $L_{P}{ }^{*}=0$ (critical models). The influence of nonscattering sources of opacity is seen to be highly important.

There are two ways in which a Kramers-like source of opacity raises the critical mass over the value yielded by pure scattering alone: (1) by directly exerting positive damping 
TABLE 2

Critical Models For THe Hydrogen MAIN SEQUence $\left(L_{P}^{*}=0\right)$

\begin{tabular}{|c|c|c|c|c|c|c|c|c|c|c|c|c|c|c|c|c|}
\hline \multirow[b]{2}{*}{ VARIABLE } & \multicolumn{4}{|c|}{$Z=0.05$} & \multicolumn{4}{|c|}{$Z=0.03$} & \multicolumn{4}{|c|}{$Z=0.01$} & \multicolumn{4}{|c|}{$Z=0.002$} \\
\hline & $\begin{array}{l}Y= \\
0.0\end{array}$ & $\begin{array}{c}Y= \\
0.200\end{array}$ & $\begin{array}{c}Y= \\
0.400\end{array}$ & $\begin{array}{c}Y= \\
0.900\end{array}$ & $\begin{array}{l}Y= \\
0.0\end{array}$ & $\begin{array}{c}Y= \\
0.200\end{array}$ & $\begin{array}{c}Y= \\
0.400\end{array}$ & $\begin{array}{c}Y= \\
0.920\end{array}$ & $\begin{array}{l}Y= \\
0.0\end{array}$ & $\begin{array}{c}Y= \\
0.200\end{array}$ & $\begin{array}{c}Y= \\
0.400\end{array}$ & $\begin{array}{c}Y= \\
0.940\end{array}$ & $\begin{array}{l}Y= \\
0.0\end{array}$ & $\begin{array}{c}Y= \\
0.200\end{array}$ & $\begin{array}{c}Y= \\
0.400\end{array}$ & $\begin{array}{c}Y= \\
0.948\end{array}$ \\
\hline $\begin{array}{l}M / M_{\odot} \ldots \ldots \ldots \\
\left(M / M_{\odot}\right) \mu^{2} \ldots \ldots \\
q_{f} \ldots \ldots \ldots \ldots \\
\beta_{c} \ldots \ldots \ldots \ldots \\
\log T_{c} \ldots \ldots \ldots \\
\log \rho_{c} \ldots \ldots \ldots \\
\rho_{c} /\langle\rho\rangle \ldots \ldots \\
\log \left(L / \dot{L}_{\odot}\right) \ldots \ldots \\
\log \left(R / R_{\odot}\right) \ldots \ldots \\
\log T_{e} \ldots \ldots \ldots \\
M_{v} \ldots \ldots \ldots \ldots \\
\omega^{2} \ldots \ldots \ldots \\
\text { Period }(h \mathrm{~h}) \ldots \ldots\end{array}$ & $\begin{array}{c}165 \\
45 \\
0.82 \\
0.50 \\
7.64 \\
0.10 \\
30 \\
6.30 \\
1.25 \\
4.71 \\
-6.7 \\
2.8 \\
9.7\end{array}$ & $\begin{array}{c}140 \\
50 \\
0.83 \\
0.47 \\
7.65 \\
0.15 \\
32 \\
6.30 \\
1.22 \\
4.73 \\
-6.6 \\
2.7 \\
9.4\end{array}$ & $\begin{array}{c}114 \\
56 \\
0.84 \\
0.45 \\
7.66 \\
0.22 \\
34 \\
6.28 \\
1.17 \\
4.75 \\
-6.4 \\
2.7 \\
9.0\end{array}$ & $\begin{array}{c}60 \\
94 \\
0.87 \\
0.37 \\
7.73 \\
0.54 \\
43 \\
6.27 \\
1.00 \\
4.83 \\
-5.8 \\
2.6 \\
7.1\end{array}$ & $\begin{array}{c}134 \\
35 \\
0.80 \\
0.54 \\
7.62 \\
0.12 \\
27 \\
6.15 \\
1.20 \\
4.70 \\
-6.4 \\
2.9 \\
8.9\end{array}$ & $\begin{array}{c}110 \\
38 \\
0.81 \\
0.53 \\
7.63 \\
0.18 \\
28 \\
6.13 \\
1.15 \\
4.72 \\
-6.2 \\
2.9 \\
8.5\end{array}$ & $\begin{array}{c}88 \\
42 \\
0.82 \\
0.51 \\
7.64 \\
0.25 \\
29 \\
6.11 \\
1.11 \\
4.74 \\
-6.0 \\
2.8 \\
8.0\end{array}$ & $\begin{array}{l}41 \\
63 \\
0.86 \\
0.43 \\
7.71 \\
0.60 \\
36 \\
6.05 \\
0.91 \\
4.82 \\
-5.4 \\
2.7 \\
6.0\end{array}$ & $\begin{array}{c}110 \\
28 \\
0.77 \\
0.59 \\
7.64 \\
0.25 \\
24 \\
6.00 \\
1.11 \\
4.71 \\
-5.9 \\
2.9 \\
7.1\end{array}$ & $\begin{array}{c}90 \\
30 \\
0.78 \\
0.57 \\
7.65 \\
0.31 \\
25 \\
5.98 \\
1.06 \\
4.73 \\
-5.8 \\
2.9 \\
6.8\end{array}$ & $\begin{array}{c}70 \\
32 \\
0.79 \\
0.56 \\
7.66 \\
0.39 \\
25 \\
5.94 \\
1.00 \\
4.75 \\
-5.5 \\
2.9 \\
6.3\end{array}$ & $\begin{array}{c}26 \\
40 \\
0.82 \\
0.52 \\
7.73 \\
0.79 \\
28 \\
5.75 \\
0.74 \\
4.83 \\
-4.5 \\
2.8 \\
4.2\end{array}$ & $\begin{array}{c}106 \\
27 \\
0.77 \\
0.60 \\
7.68 \\
0.39 \\
23 \\
5.97 \\
1.05 \\
4.73 \\
-5.7 \\
2.8 \\
5.9\end{array}$ & $\begin{array}{c}86 \\
28 \\
0.78 \\
0.58 \\
7.69 \\
0.46 \\
23 \\
5.94 \\
1.00 \\
4.75 \\
-5.5 \\
2.8 \\
5.6\end{array}$ & $\begin{array}{c}64 \\
29 \\
0.78 \\
0.58 \\
7.70 \\
0.54 \\
24 \\
5.87 \\
0.93 \\
4.77 \\
-5.2 \\
2.9 \\
5.1\end{array}$ & $\begin{array}{c}21 \\
32 \\
0.79 \\
0.57 \\
7.76 \\
0.96 \\
25 \\
5.59 \\
0.63 \\
4.85 \\
-4.0 \\
2.9 \\
3.2\end{array}$ \\
\hline
\end{tabular}


through the behavior of the temperature and density exponents of the opacity law and (2) by indirectly increasing the central condensation of the star.

The first effect requires no discussion since it has been abundantly reviewed in the literature. With regard to the second effect, dimensional arguments show that an opacity source which decreases inward into the star leads to an expanded radius; the mean density is therefore lowered while the central density remains practically fixed by the nuclear-reaction rate. The necessity of a higher radiation pressure to compensate the higher central condensation (which, between them, determine the size of the pulsation amplitudes in the energy-producing region) requires a larger mass.

The two foregoing effects of variable opacity are easily discernible in Tables 2 and 3 by observing that, for constant $\mu, M_{\text {erit }} / M_{\odot}$ increases with increasing $Z, \rho_{r} /\langle\rho\rangle$, and $1-\beta_{r .}$. In this connection, it is interesting, but not surprising, to note that $\omega^{2}$ is nearly

TABLE 3

Critical MOdels For THE Helium MAIN SEQUENCE $\left(L_{P} *=0\right)$

\begin{tabular}{|c|c|c|c|}
\hline \multirow[b]{2}{*}{ VARIABLE } & \multicolumn{3}{|c|}{$Z$} \\
\hline & 0.05 & 0.02 & 0.0 \\
\hline 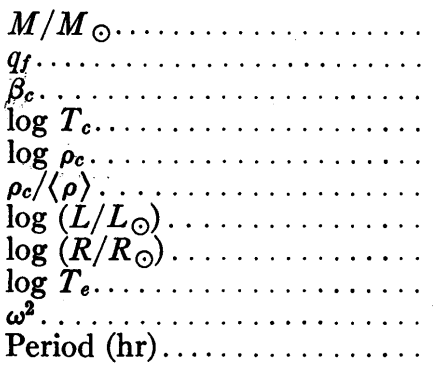 & $\begin{array}{l}19 \\
0.76 \\
0.54 \\
8.30 \\
2.58 \\
33 \\
5.64 \\
0.12 \\
5.11 \\
3.5 \\
0.52\end{array}$ & $\begin{array}{l}15 \\
0.72 \\
0.60 \\
8.28 \\
\cdot 2.64 \\
28 \\
5.44 \\
0.04 \\
5.11 \\
3.4 \\
0.45\end{array}$ & $\begin{array}{l}13 \\
0.70 \\
0.63 \\
8.28 \\
2.68 \\
25 \\
5.33 \\
-0.01 \\
5.10 \\
3.3 \\
0.41\end{array}$ \\
\hline
\end{tabular}

constant for all our models, confined to the narrow range 2.6-2.9 for the hydrogen-burning models and 3.3-3.5 for the helium-burning models. We have pointed out elsewhere (Simon and Stothers 1969) that this constancy is a general phenomenon of nuclearenergized pulsation $\left(\omega_{\text {crit }}{ }^{2} \sim 3\right)$; in fact, this characteristic helped us considerably in locating the critical masses of the present paper.

\section{a) Hydrogen-Burning Models}

Two outstanding features appear in connection with the chemical composition of the hydrogen-burning models. First, the well-known trend of the critical mass with mean molecular weight (the chief effect of $X$ and $Y$ ) is plainly exhibited. However, $\left(M_{\text {erit }} /\right.$ $\left.M_{\odot}\right) \mu^{2}$ is not a constant, as it is for homologous stars "built on the same model." This leads to examination of the second feature, namely, the extraordinary sensitivity of the critical mass to nonscattering sources of opacity in the envelope. Bound-free absorption by metals is the dominant source when $Z>0.03$, as $\left(M_{\text {crit }} / M_{\odot}\right) \mu^{2}$ for these models is closer to Ledoux's value of $\sim 100$ than to Schwarzschild and Härm's value of 21 . However, even when $Z$ is reduced essentially to zero, the opacity does not go over into pure electron scattering, on account of the contribution from free-free absorption by hydrogen and helium; therefore, $\left(M_{\text {crit }} / M_{\odot}\right) \mu^{2}$ never becomes as low as the uniform value calculated by Schwarzschild and Härm for the pure-scattering case. The computations of 
$\left(M_{\text {crit }} / M_{\odot}\right) \mu^{2}$ for constant $Z$ show that the helium opacity is slightly more important than the hydrogen opacity in determining the critical mass.

\section{b) Helium-Burning Models}

The effect of the helium opacity is equally striking in the hotter and denser models for the helium-burning stars. This effect alone approximately doubles the critical mass found earlier by Boury, Ledoux, and Noels-Grötsch (7-9 $\left.M_{\odot}\right)$.

Evolution during core helium burning turns out also to have a stabilizing effect, which we have assessed by calculating the critical mass for models with $Z=0$ in the envelope, $Z=0.5$ in the core, and a chemical discontinuity between envelope and core. Such a structure does represent a realistic stage in the evolution because the convective core of helium-burning stars increases monotonically in time. For simplicity, we have used the triple- $a$ process as the energy source at this stage of evolution, although, correctly, some of the energy production will come from ${ }^{12} \mathrm{C}(a, \gamma){ }^{16} \mathrm{O} .{ }^{1}$ The critical mass is found to be $45 M_{\odot}$, which is much larger than the critical mass for the initial homogeneous stars because the reduction in temperature sensitivity of the nuclear reactions and the increase of central condensation overcompensate the increase of radiation pressure and the reduced effect of atomic absorption on the damping. The pulsational characteristics of the model are $\omega^{2}=2.9$ and Period $=0.84$ hour. Our result indicates that evolution rapidly stabilizes helium stars just as it does hydrogen stars (see Schwarzschild and Härm [1959] for evolution during hydrogen burning).

\section{DISCUSSION OF APPROXIMATIONS}

Four important approximations have been made in our work. It is useful to try to estimate the effect of each on the critical mass. Two are concerned with destabilization in the core and two with damping in the envelope.

1. In the hydrogen-burning models, we made the simplifying approximation that $\nu_{\text {eff }}=\nu$. Reducing $\nu_{\text {eff }}$ by a unit results in an increase of the critical mass by typically $\sim 3$ percent (but this is dependent somewhat on the mass).

2. A more accurate procedure than the Schwarzschild-Härm approximation for evaluating luminosity amplitudes in the convective core should take account of nonadiabatic effects. A step in this direction has been made by Boury, Gabriel, and Ledoux (1964), with the following result: The assumption of adaptive convection results in essentially no modification at all, whereas the assumption of nonadaptive convection provides some additional destabilization, reducing the critical mass by at most 10 percent.

3. Nonadiabatic effects on the envelope pulsation have been estimated by the procedure described in the Appendix. Our complete neglect of such effects in the hydrogen-burning models leads to an overestimation of the positive damping, and hence of the critical hydrogen-burning masses, by $\sim 10$ percent. ${ }^{2}$ The accuracy of our estimation can probably be considered fair because of the very narrow extent of the uncertain transition region-in all of the models of Tables 2 and 3 it covers $\sim 2$ percent of the radius and less than $10^{-4}$ of the stellar mass.

4. Our neglect of running waves in the atmosphere is more difficult to assess since the star may also be ejecting matter. In the coarse approximation of Schwarzschild and Härm (1959), the inclusion of running waves would increase the critical mass by $\sim 4$ percent in all cases, but this estimate should not be considered reliable.

Since the uncertainties in $\nu_{\text {eff }}$ and running waves on the one hand, and in nonadaptive convection and outer-envelope damping on the other hand, tend to cancel each other,

1 The error incurred by our simplification is small, since the temperature exponents for the two processes are nearly equal at the relevant central temperature.

${ }^{2}$ For pure-scattering models, the overestimation is $\sim 5$ percent. In contrast, a standard adiabatic treatment of the present helium-burning models would allow negative damping to dominate in the outer envelope and thereby lower the critical helium-burning mass to about $5 M_{\odot}$ (for $Z=0$ ). 
the formal error in our models might seem slight. However, the surface regions are still poorly treated, and the unknown effect of inaccuracies in the opacity exponents $a$ and $\eta$ do not permit us to claim any great precision in the critical masses of Tables 2 and 3.

\section{v. CONCLUSTON}

An interesting possibility arises of fixing the $Y$ and $Z$ content of extreme Population I stars by combining our theoretical results with observations of stars at the very top of

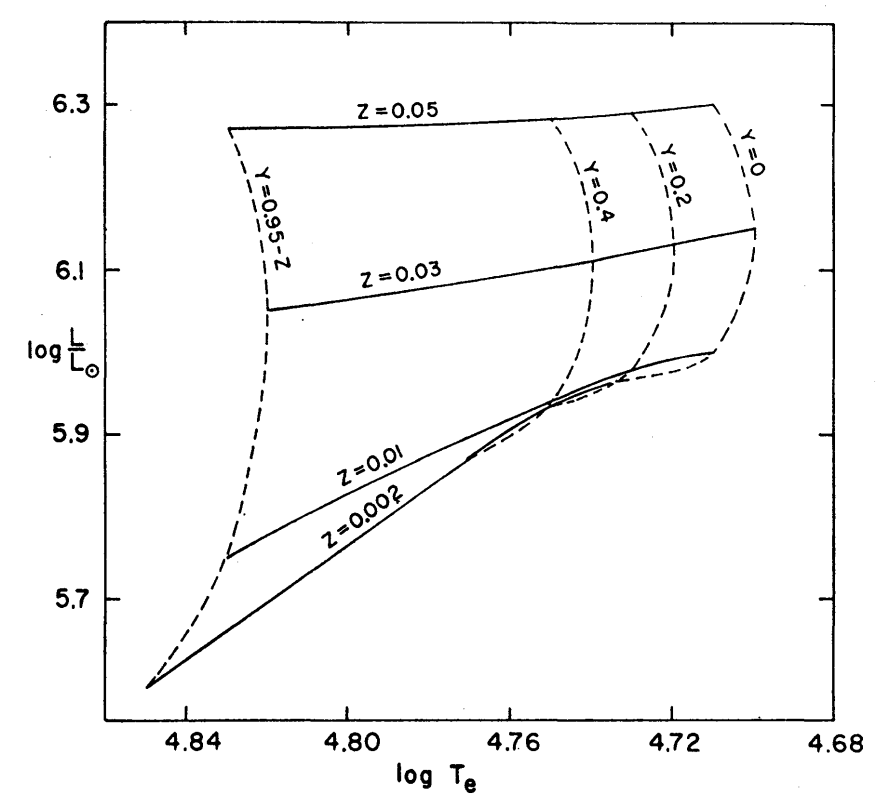

Fig. 1. - Theoretical H-R diagram for models representing the maximum mass of pulsationally stable, homogeneous main-sequence stars. Curves are labeled with the helium $(Y)$ and metals $(Z)$ content.

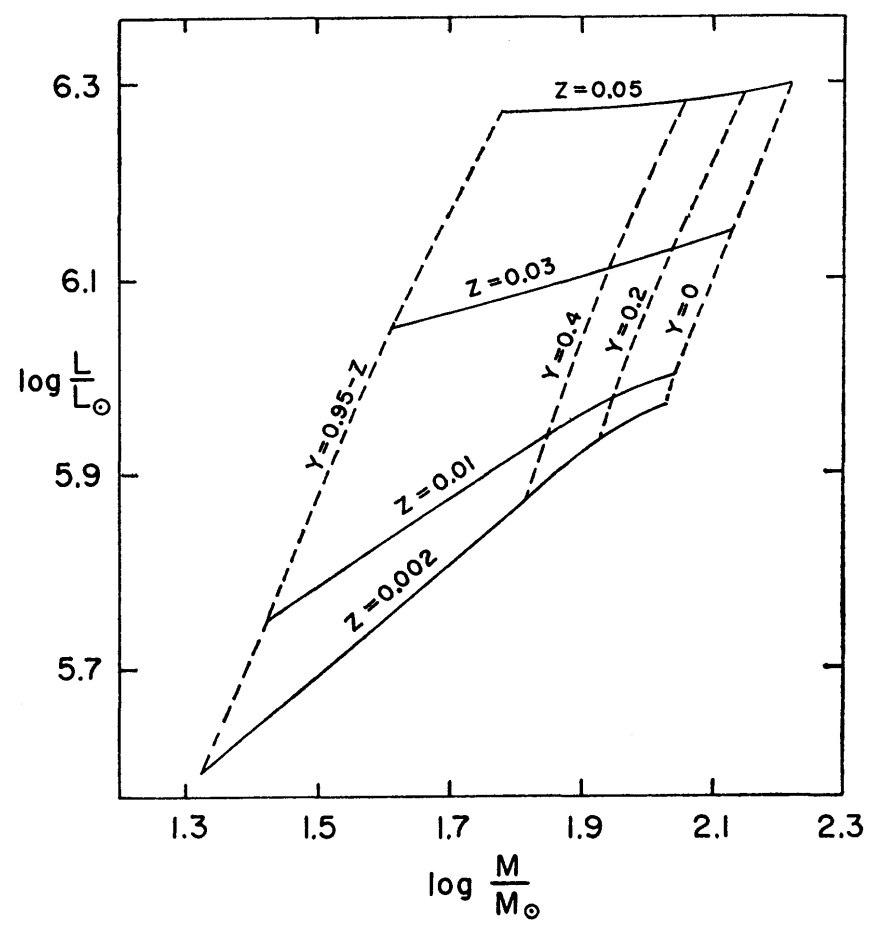

Fig. 2.-Theoretical mass-luminosity diagram for the models of Fig. 1 
the main sequence. These observations may consist of (1) the temperature and luminosity of the tip of the main sequence, (2) periodic variations in light and radial velocity of the brightest stars, and (3) masses of the brightest binary stars. Figure 1 displays our predictions for the main sequence on the $H-\mathrm{R}$ diagram. It is apparent that the luminosity is affected far more by $Z$ than by $Y$, whereas the effective temperature has the opposite sensitivity. However, the narrowness of the ranges in luminosity and effective temperature does not provide much hope of being able to use them to fix the chemical composition. Figure 2 shows our predictions in the mass-luminosity plane. Unfortunately, the requisite observational data are, at present, too sparse or unreliable to make a detailed comparison with our theoretical results.

If one simply adopts the best current estimates for the chemical composition of extreme Population I, $0.2 \leq Y \leq 0.4$ and $0.02 \leq Z \leq 0.04$ (Morton 1968), then the critical mass is expected to lie in the range 80-120 $M_{\odot}$.

N. R. S. acknowledges the support of an NAS-NRC research associateship under the National Aeronautics and Space Administration.

\section{APPENDIX}

\section{CALCULATION OF NONADIABATIC EFFECTS IN THE OUTER ENVELOPE}

Criteria for determining the importance of nonadiabatic contributions to the pulsational damping integral have been discussed, for example, by Ledoux and Walraven (1958) and by Ledoux and Whitney (1961). In these discussions the star is divided into three regions: (1) $r>r^{*}$, where the heat capacity of the gas is so small that the pulsation has virtually no effect on the luminosity; (2) $r<r^{\dagger}$, where the pulsation is essentially adiabatic; and (3) the transition region $r^{*}>r>r^{\dagger}$, where nonadiabatic terms can make important contributions. To use these criteria in practice, we have adopted the following prescription.

The point $r^{*}$ may be fixed roughly by considering the ratio

$$
\psi=\frac{\int_{M\left(r^{*}\right)}^{M} \delta U d M(r)}{L(\operatorname{Period} / 2 \pi)},
$$

where $L$ is taken to be the equilibrium luminosity (constant in the envelope), $U$ is the internal energy of the matter and radiation, and $\delta U$ is the change in $U$ over a quarter-period of the pulsation. It is sufficient for our purposes to use the adiabatic temperature amplitude to evaluate the change in $U$,

$$
\delta U=\frac{P}{\rho}\left(\frac{24-21 \beta}{8-6 \beta}\right)\left(\frac{\delta T}{T}\right)_{a}
$$

Since the denominator in the expression for $\psi$ gives approximately the energy fed by the flux into mass $M-M\left(r^{*}\right)$ in the same quarter-period, the ratio $\psi$ measures the ability of the layers in question to alter the flux via their pulsation. As we go inward from the stellar surface, as long as $\psi$ remains small the luminosity will be unaffected by the pulsation and we may take $\delta L(r)=$ constant. On the other hand, when some point $r=r^{*}$ is reached, $\psi$ becomes sizable and the transition region begins.

As one goes farther into the star, the transition region extends down to the point $r=r^{\dagger}$, where nonadiabatic contributions to the pulsation become small. We may approximately fix the beginning of the adiabatic region by considering the ratio $\phi$ of the nonadiabatic part to the adiabatic part of the temperature amplitude,

$$
\phi=\frac{(\delta T)_{n}}{(\delta T)_{a}}=\left(\frac{2}{24-21 \beta}\right) \frac{\rho}{P} \frac{d}{d M(r)}\left(\frac{\delta L}{L}\right) \frac{L(\operatorname{Period} / 2 \pi)}{(\delta T / T)_{a}} .
$$

The pulsation may be said to be adiabatic when this ratio becomes sufficiently small. 
In calculating models, we have proceeded as follows: (1) the amplitudes are taken throughout to be the adiabatic ones, with the adiabatic boundary conditions described in the main text; (2) the transition region is taken to extend from $r^{*}=r(\psi=0.01)$ to $r^{\dagger}=r(\phi=0.01)$; and (3) the true contribution from this region to the damping integral is taken to be half the value given by the adiabatic calculation, i.e.,

$$
\frac{L_{\mathrm{PH}}}{L}=\frac{1}{2} \int_{M(r \dagger)}^{M\left(r^{*}\right)} \frac{d}{d M(r)}\left(\frac{\delta L}{L}\right)_{a}\left(\frac{\delta T}{T}\right)_{a} d M(r)+\int_{0}^{M\left(r^{\dagger}\right)} \frac{d}{d M(r)}\left(\frac{\delta L}{L}\right)_{a}\left(\frac{\delta T}{T}\right)_{a} d M(r) .
$$

Since the true amplitudes will approach the adiabatic ones for $r<r^{\dagger}$, our first assumption will provide an accurate evaluation of the damping in the adiabatic region. In the transition region, the adiabatic amplitudes will always overestimate the damping. Thus the maximum contribution from this region is the adiabatic value, while the minimum contribution is obviously zero. By adopting a value halfway between these extremes, we reduce the uncertainty. The limiting values we have chosen for $\psi$ and $\phi$ give the transition region a rather generous extent. However, in the stellar models considered here, this region turns out to be thin enough that the net damping it contributes is small compared with the whole damping of the star.

\section{REFERENCES}

Baker, N., and Kippenhahn, R. 1965, Ap. J., 142, 868.

Boury, A. 1963, Ann. d'ap., 26, 354.

. 1964, Ap.J., 140, 1322 .

Boury, A., Gabriel, M., and Ledoux, P. 1964, Ann. d'ap., 27, 92.

Boury, A., and Ledoux, P. 1965, Ann. d'ap., 28, 353.

Christy, R. F. 1966, Ap.J., 144, 108.

Cimino, M., Giannone, P., Giannuzzi, M. A., Masani, A., and Virgopia, N. 1964, Nuovo Cimento, 33, 663.

Cox, J. P. 1955, A p. J., 122, 286.

Cox, A. N., and Stewart, J. N. 1965, Ap. J. Suppl., 11, 22.

Deinzer, W., and Salpeter, E. E. 1965, A p. J., 142, 813.

Ledoux, P. 1941, Ap. J., 94, 537.

Ledoux, P., and Boury, A. 1960, Mém. Soc. R. Sci. Liège, Ser. 5, 3, 298.

Ledoux, P., and Walraven, Th. 1958, Hdb. d. Phys., 51, 353.

Ledoux, P., and Whitney, C. A. 1961, Nuovo Cimento Suppl., Ser. 10, 22, 131.

Masevich, A. G., Kotok, E. V., Dluzhnevskaya, O. B., and Masani, A. 1965, Astr. Zh., 42, 334.

Morris, S. C., and Demarque, P. 1966, Zs. f. A p., 64, 238.

Morton, D. C. 1968, A p. J., 151, 285.

Noels-Grötsch, A. 1967, Ann. d'ap., 30, 349.

Perdang, J. 1965, Bull. Soc. Roy. Sci. Liège, 34, 736.

Reeves, H. 1965, in Stellar Structure, ed. L. H. Aller and D. B. McLaughlin (Chicago: University of Chicago Press), p. 113.

Rosseland, S. 1949, The Pulsation Theory of Variable Stars (Oxford: Clarendon Press).

Schwarzschild, M. 1958, Structure and Evolution of the Stars (Princeton: Princeton University Press).

Schwarzschild, M., and Härm, R. 1959, A p. J., 129, 637.

Simon, N. R., and Stothers, R. 1969, A p. J., 156, 377.

Traving, G. 1966, Abundance Determinations in Stellar Spectra (IAU Symp. No. 26), ed. H. Hubenet (London: Academic Press), p. 213. 
